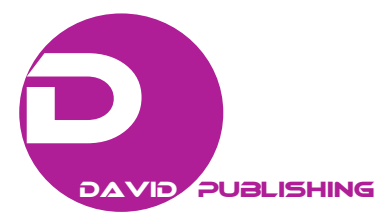

\title{
Management of Maxillary Ameloblastoma with Vascularized Bone Graft
}

\author{
Siska and Iwan Tofani \\ Department of Oral and Maxillofacial Surgery, Faculty of Dentistry, University of Indonesia, Jakarta 10430, Indonesia
}

\begin{abstract}
Maxillary ameloblastoma is quite rare especially the one that outcomes facial deformity. Eighty-five percent of ameloblastoma cases happen to the mandible, mostly in the molar-ascending area. About $15 \%$ of ameloblastomas occur in maxilla. We are reporting a maxillary ameloblastoma case of an Indonesian patient. Bilateral Weber Ferguson approach was performed to facilitate total maxillectomy and continued by fibular graft reconstruction. The Weber Ferguson provides a wide access to all areas of the maxilla and orbital floor. From three month post operative evaluation we achieved favourable aesthetic and functional results for patient.
\end{abstract}

Key words: Maxillary ameloblastoma, bilateral Weber Ferguson, fibular graft reconstruction.

\section{Introduction}

Ameloblastoma is an odontogenic tumour arising from residues of the dental lamina. The occurrence of ameloblastoma is in the ages of 30's and 40's, both women and men are afflicted equally. Slow development and swelling without pain become the characteristics of ameloblastoma that lead to the widening of cortical bone, the perforation of lingual or buccal plate and infiltration to the soft-tissue. Ameloblastoma of maxilla is particularly dangerous because of the difficulty of obtaining an adequate surgical margin of the tumor [1].

The treatment of ameloblastoma is proceeded by physical examination extra orally to know whether the case is symmetry or asymmetry. Before radiography examination is done, aspiration is carried out to get histopathological findings. Simple and small size ameloblastoma can be treated by simple enucleation and curettage to en bloc resection [2]. Since the radiography examination cannot show clear margin of the tumour, it is recommended to apply Carnoy's solution to the doubt area. Large size ameloblastoma should be treated by marginal resection. Many

Corresponding author: Iwan Tofani, DDS, OMFS, Ph.D., professor, research field: oral surgery. techniques have been developed to access large size tumor in the maxilla. One of the most commonly used trans-facial approaches to the mid-face for the resection of maxillary tumor is the Weber Ferguson maxillectomy incision [3]. Weber Ferguson provides a wide access to all areas of the maxilla and orbital floor. This incision gives great visualization for surgeon and causes very little deformity [4].

Reichart et al. [5] suggested that histological sub-types may have prognostic implications for recurrence; they are the follicular that has the highest rate of recurrence $(29.5 \%)$, the plexiform that constitutes $16.7 \%$ and the acanthomatous that has only $4.5 \%$. Careful attention must be given to long-term post-operative follow up.

This case reported present ameloblastoma in maxilla that is treated by total maxillectomy with Weber Fergusson technique and vascularized fibular graft reconstruction.

\section{Case Report}

An Indonesian woman, 49 years old was referred to Cipto Mangunkusumo Hospital, Jakarta with chief complaint of swelling in her upper gum and lip two months before admission. She was treated only using antibiotics and there was no reaction before admission. 
She experienced no pain, no blood discharge or weight loss, but had history of hypertension, heart and lung diseases. The patient took Lianopril, Amlodipin, and Bisoprolol as her routine medication.

Based on extra oral examination, there was an asymmetry in the maxilla (Fig. 1).

The intraoral examination showed a large mass in the upper jaw approximately $6 \times 5 \times 5 \mathrm{~cm}$ in size, well defined border, smooth mass in the maxilla (Fig. 2).

The panoramic radiograph showed a radiolucent lesion in the midline maxilla, approximately $25 \mathrm{~mm}$ in size, well-defined border in superior (nasal) extending to proximal. The lesion affected the anterior maxilla, septum, and nasal (Fig. 3).

CT scans result showed a lesion in the maxilla extending from regio 16-26 to two-thirds of nasal foramen. The lesion border was well-defined with irregular shape and an appearance of scalloped pattern (Fig. 4).

The laboratory findings showed that the patient had quite low APTT (Activated Partial Thromboplastin
Time) and high SGOT-SGPT (Serum Glutamic Oxaloacetic Transaminase-Serum Glutamic Pyruvic Transaminase). A reddish brown liquid was obtained from the needle aspiration.

From the records of history and examination, we came to the diagnose of ameloblastoma. We performed initial incisional biopsy and it confirmed the diagnose, plexiform and follicular ameloblastoma. There were palisading columnar cells in its side and metaplastic squamous epithelial cells in the center. Stroma contained of fibro collagen tissue and there were necrotic bone and inflammation cells in tumor margin. Therefore, we did the operation in the maxilla with the Bilateral Weber Ferguson approach and the operation was continued by Plastic Surgery team to reconstruction using vascularized graft with fibular graft maxillary resection and vascularized bone graft subsequently (Fig. 5).

It was taken from free fibular flaps (cruris sinistra left foot). The result of the operation was good and achieved favourable aesthetic (Fig. 6).
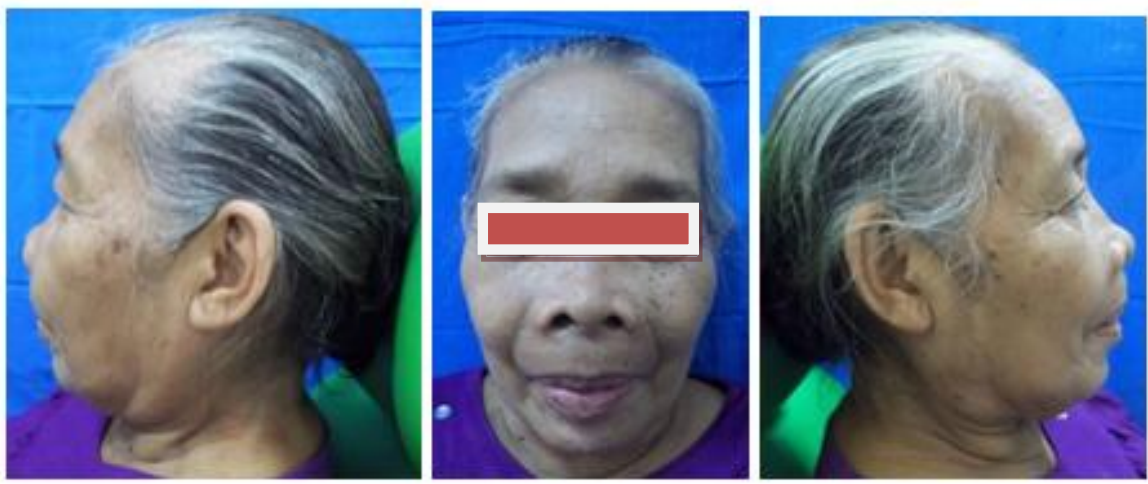

Fig. 1 Extra Oral Examination.

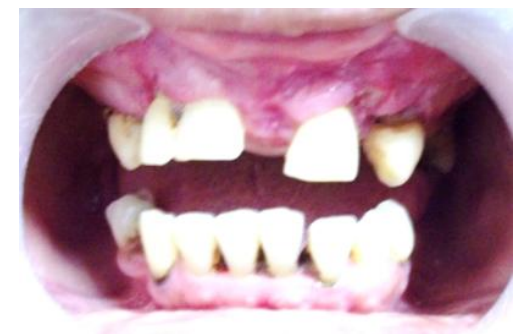

Fig. 2 Intra Oral Examination. 
Fig. 3 Panoramic Radiograph.
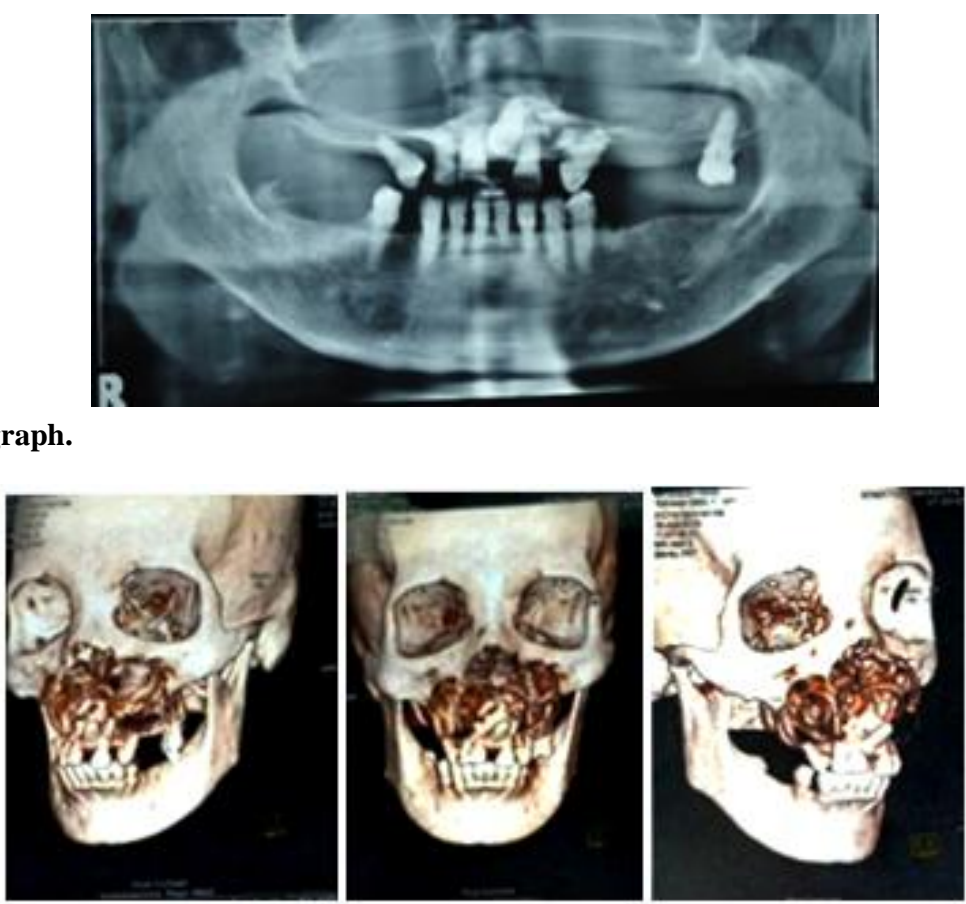

Fig. 4 3D Reconstruction.
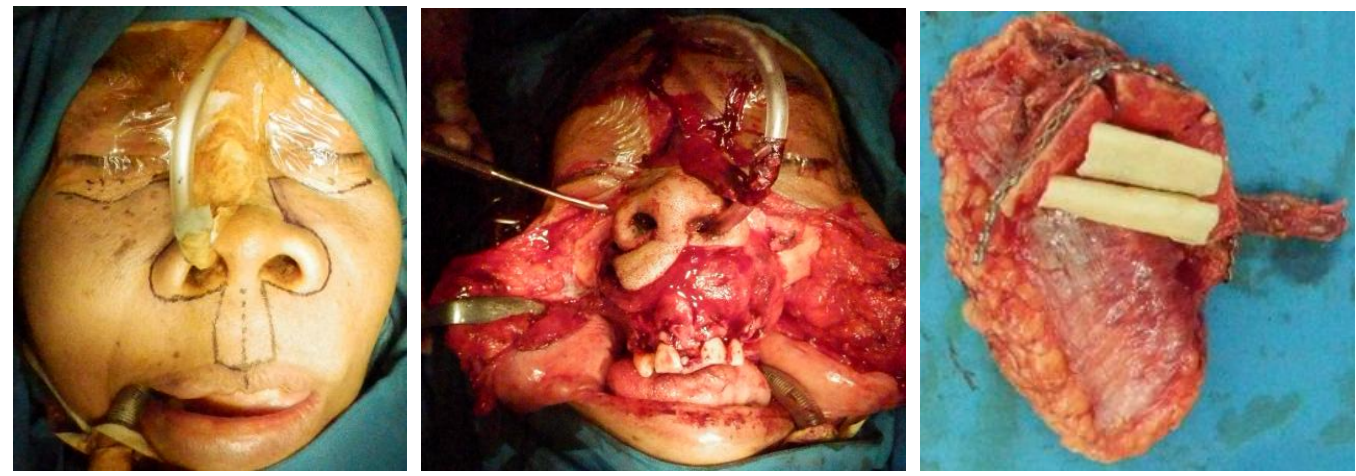

Fig. 5 Design Weber Ferguson and vascularized bone graft.

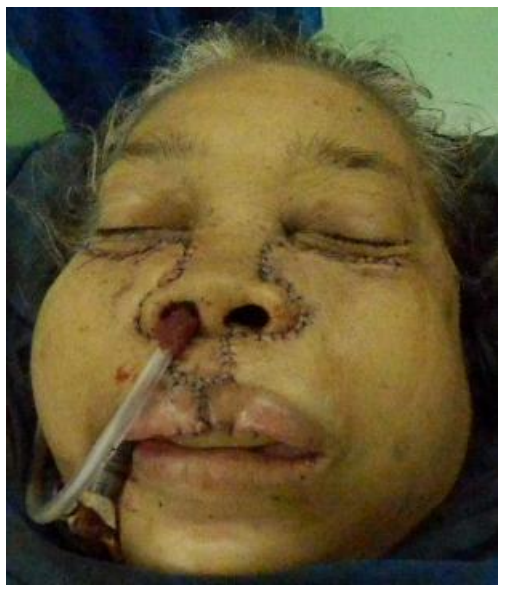

Fig. 6 Post Operative. 


\section{Discussion}

Ameloblastoma is thought to be an odontogenic tumor which is commonly found in the mandible and maxilla. In this case, the patient had maxillary ameloblastoma. This kind of tumor usually occurs in the ages around 30s and 40s, and the patient in this case was in 49 years old. From the histopathological examination, a case of ameloblastoma can be classified as plexiform and follicular ameloblastoma. The treatments that can be applied for ameloblastoma are radical approaches. The surgical approach is dependent on the anatomic location, extent, and histology of the tumor. Malignant tumors of the maxilla including tumors of the upper gum, hard palate, or floor of maxillary antrum, can be adequately excised by a transoral partial maxillectomy [5]. There are many types of surgical approach to access maxilla, such as maxillary vestibular approach, midfacial degloving approach, and Weber Ferguson approach [6].

The Weber Ferguson approach is indicated as an approach to get access to the tumors in the maxilla extending superiorly to the infra orbital nerve and into or involving the orbit. It provides a wide access to all areas of the maxilla and orbital floor. Tarsorrhaphy sutures are placed in the eyelids. The incision is made through skin and subcutaneous tissue along the nose. The full thickness upper lip is transected and the labial artery are ligated or coagulated. The procedure then extends sub labially along the mucobuccal fold preserving as much mucosa as possible, up to the maxillary tuberosity. The subciliary component extends through the orbicularis oculi muscle and then down to bone in the preseptal plane. The cheek flap is elevated off the maxilla to its lateral border in a subperiosteal plane with electrocautery. A supra periosteal dissection plane will be necessary in the subcutaneous tissues if there is tumor invasion of the antero lateral maxillary wall. In most cases, the infraorbital nerve is sacrificed to facilitate tumor removal. After the tumor removal, the orbicularis oculi muscle is approximated with absorbable sutures. The subcutaneous tissues are also closed with absorbable sutures, as is the orbicularis oris muscle. The vermillion border is reapproximated accurately and the skin is closed with fine nylon sutures [6].

The marking of the ameloblastoma border clearly can be done with cauterization by adding minimum 1 $\mathrm{cm}$ around the border of ameloblastoma. The decision to treat ameloblastoma cannot be carried out only with simple enucleation because of the relapse or recurrence of this tumor. Several systematic reviews of ameloblastoma do not recommend enucleation or dredging method. Due to high recurrence rate of maxillary ameloblastoma, some surgeons have suggested using only an obturator for the defect left after resection. However, a secondary operation must be performed whenever recurrence is back. Weber-Ferguson's approach provides a very good exposure to the maxillary region $[7,8]$. This incision gives more direct access to the maxilla and allows more unimpeded resection of tumor. This approach will avoid resection of the uninvolved structures owing to its direct access to the pathologies of the maxilla $[9,10]$. The approach is more flexible with relatively minimum number of complications such as scar formation and lymph edema [11]. In this case, the patient was treated by extra oral incision Bilateral Weber Ferguson and resection combined with vascularized bone graft.

The patient was resected because the tumor had already invaded the retro maxillary space. The resection was carried out by removing the entire maxillary sinus and lateral wall of the nose. Bone graft was treated to the patient for the purpose of jaw reconstruction and to restore blood circulation after vascularized graft method was performed.

\section{Conclusion}

Ameloblastoma as an odontogenic tumor can occur in the maxilla although it more frequently happens in the mandible. In this case report, we presented an ameloblastoma in the maxilla of 49-year-old woman 
and the biopsy confirmed a plexiform follicular ameloblastoma. Bilateral Weber Ferguson approaches is considered very flexible and suitable for the treatment of complex pathologies of mid face region. The case was treated using maxillary resection and reconstructed with subsequent vascularized bone grafted as the treatment of choice.

\section{Acknowledgment}

Thanks to Dwi Ariawan, DDS,MARS,OMFS and Vera Julia, DDS, OMFS and the team plastic surgery for the provision of data that we present in this case.

\section{References}

[1] Neville, B. W., Damm, D. D., Allen, C. M., and Chi, A. C. 2015. Oral and Maxillofacial Pathology. Philadelphia: Elsevier.

[2] Navarro, C. M., Principi, S. M., Massucato, E. M. S., and Sposto, M. R. 2004. "Maxillary Unicystic Ameloblastoma.” Dentomaxillofacial Radiol. 33 (1): 60-2.

[3] Fernandes, R., and Ord, R. 2006. "Access Surgery for Oral Cancer.” Oral Maxillofac Surg. Clin. North Am. 18 (4): 565-71.
[4] Boonsiriseth, K., Kiattavorncharoen, S., Thungrat, A., and Natthamet, W. 2015. "The Incidence of oro-Maxillofacial Lesions (10 Years) in the Department of Oral and Maxillofacial Surgery, Mahidol University: Ameloblastoma." Mahidol. Dent. J. 35 (1): 21-30.

[5] Reichart, P. A., Philipsen, H. P., and Sonner, S. 1995. “Ameloblastoma: Biological Profile of 3677 Cases." Eur. J. Cancer Part B Oral Oncol. 31 (2): 86-99.

[6] Bartlett, S., Ehrenfeld, M., Mast, G., and Sugar, A. 2012. Congenital Deformities. Accessed January 1, 2012. https://www2.aofoundation.org/wps/portal/surgery?bone $=\mathrm{CMF} \&$ segment $=$ Congenital\&showPage $=$ approach .

[7] Motiee-Langroudi, M., Harirchi, I., Amali, A., and Jafari, M. 2015. "Reconstruction of Midface and Orbital Wall Defects after Maxillectomy and Orbital Content Preservation with Titanium Mesh and Fascia Lata: 3-Year Follow-Up.” J. Oral Maxillofac. Surg. 73 (12): 2447.

[8] Bhavana, K., Tyagi, I., and Ramani, M. K. 2012. "Modified Incision for Maxillectomy: Our Experience." Indian J. Otolaryngol. Head Neck Surg. 64 (2): 184-7.

[9] Dediol, E., Uglešić, V., Zubčić, V., and Knežević, P. 2013. "Brown Class III Maxillectomy Defects Reconstruction with Prefabricated Titanium Mesh and Soft Tissue Free Flap.” Ann. Plast. Surg. 71 (1): 63-7.

[10] McCarthy, C. M., and Cordeiro, P. G. 2010. "Microvascular Reconstruction of Oncologic Defects of the Midface.” Plast Reconstr Surg. 126 (6): 1947-59. 